\title{
Learning Support Assessment Study of a Computer Simulation for the
}

\section{Development of Microbial Identification Strategies}

\author{
TRISTAN E. JOHNSON*1 AND CLARK GEDNEY ${ }^{2}$ \\ Educational Technology, Department of Curriculum and Instruction, ${ }^{1}$ and BioMedia Center for Instructional \\ Design, Department of Biological Sciences, ${ }^{2}$ Purdue University, West Lafayette, Indiana 47907
}

This paper describes a study that examined how microbiology students construct knowledge of bacterial identification while using a computer simulation. The purpose of this study was to understand how the simulation affects the cognitive processing of students during thinking, problem solving, and learning about bacterial identification and to determine how the simulation facilitates the learning of a domain-specific problem-solving strategy.

As part of an upper-division microbiology course, five students participated in several simulation assignments. The data were collected using think-aloud protocol and video action logs as the students used the simulation. The analysis revealed two major themes that determined the performance of the students: Simulation Usage—how the students used the software features and Problem-Solving Strategy Development—-the strategy level students started with and the skill level they achieved when they completed their use of the simulation.

Several conclusions emerged from the analysis of the data: (i) The simulation affects various aspects of cognitive processing by creating an environment that makes it possible to practice the application of a problem-solving strategy. The simulation was used as an environment that allowed students to practice the cognitive skills required to solve an unknown. (ii) Identibacter (the computer simulation) may be considered to be a cognitive tool to facilitate the learning of a bacterial identification problem-solving strategy. (iii) The simulation characteristics did support student learning of a problem-solving strategy. (iv) Students demonstrated problem-solving strategy development specific to bacterial identification. (v) Participants demonstrated an improved performance from their repeated use of the simulation.

Due to the increased accessibility of technology, researchers are studying the impact of the computer in developing higher-order thinking skills. Computers are thought to have great potential for assisting the development of problem-solving skills (9). Research on computer-assisted instruction and simulations suggests that computers are effective in helping students reach deeper understandings of information being learned (6). Quinn (personal communication) showed that simulations enhance students' problem-solving skills by providing them a practice environment in which they can refine their higher-order thinking strategies. Other studies have also shown instructional benefits of using computer simulations for development of higher-order thinking strategies $(2-4,5,7,8)$.

Computer-based instructional simulations are computer programs that contain a manipulatable model of a real or theoretical system (8). Simulations typically focus on a learning environment without taking control from the learner. As a result, simulations permit the attainment of cognitive learning goals beyond traditional and other computer-based methods $(3,8)$.

There is a general belief that computer simulations are useful tools to help develop problem-solving skills. Past research has suggested several generic instructional benefits of using instructional computer simulations (1). Yet in re-

\footnotetext{
*Corresponding author. Mailing address: Educational Technology, Department of Curriculum and Instruction, 1442 Liberal Arts and Education Building, Purdue University, West Lafayette, IN 47907-1442. Phone: (765) 496-6367. Fax: (765) 496-1622. Email: tj@purdue.edu.
}

gards to learning problem-solving strategies for bacterial identification, there is no empirical data supporting the usefulness of simulations, nor is there data explicating how computer simulations are useful, nor descriptions of how more useful simulations can be designed.

The focus of this paper is to present findings and results of a study that investigated how a computer simulation supported student learning. To understand this, we present data and results that answer the following questions. How do students use Identibacter to practice bacterial identification? And, how do the simulation components support student learning of a bacterial identification problem-solving strategy?

After we present a brief description of the computer simulation, we will delineate the research methods, present the findings, and discuss the results of this study as they relate to the key simulation characteristics that support student learning.

\section{DESCRIPTION OF SIMULATION}

In response to instructional needs of an introductory undergraduate microbiology course, a team of researchers at the BioMedia Center for Instructional Design at Purdue University developed the computer simulation, Identibacter interactus (Identibacter). The first version of the simulation (Identibacter) is available through McGraw-Hill. The second version (BacteriaID) includes revisions based on this study. This enhanced version is available through BenjaminCummings. For more information about BacteriaID go to the following URL, http://biomedia.bio.purdue.edu/ 
BacteriaID/. This cognitive tool was designed to provide a means for students to practice the cognitive skills utilized in the identification of microorganisms. Identibacter has a database that comprises 57 different chemoheterotrophic bacterial species. Most of these are species that are commonly used in introductory microbiology laboratory courses, but the program also includes 16 human pathogens that would be difficult to use in a traditional laboratory setting. With the simulation, students can run 51 different diagnostic tests.

Identibacter's learning environment provides several key support components. Overall, the computer software simulates the collection of experimental data and has reference information that is fundamental to the identification of an unknown bacterial culture. The first major component is the Test Window. From this window, the user is presented with the unknown organism. The student determines what test to run and then the simulation displays the test results. The test categories include the following types of tests: morphology, hydrolysis, metabolism, enzyme, fermentation, and carbon growth source. Each category provides specific tests that students can run. For example, students may run several morphological type tests such as Gram reaction, phase contrast, motility, and endospore heat.

The second major component is the Help feature. During the identification process, students may decide to use several simulation support components. Identibacter provides two types of online support. Reference-only help simply provides information. This includes information such as a list of unknown species, details about each possible test, and a list of user test interpretations. The second type of help is interactive reference help. This component provides taxon characteristics information and a mechanism for the management of group and genera information.

\section{METHODS}

The research was designed to look at two main facets: students' general use of the software and students' strategy development processes. The research emphasis was on the subjective learning experiences of several individuals. This focus led to the primary purpose of this study, to better understand the effects of the computer simulation on the cognitive development of domain-specific problem-solving strategies.

By analyzing the work of five microbiology students, there was an attempt to determine how the participants constructed knowledge of bacterial identification while using Identibacter. More specifically, the analysis process sought to understand how students used Identibacter to practice bacterial identification and what, if any, components of the simulation were useful in supporting the learning process that students went through as they identified bacteria.

Ultimately, this study was designed to understand the effectiveness of the simulation by looking into students' constructions of knowledge, what they learn from the simulation, and what they experience. From this, propositions were created to explain what the data were telling the researchers about the learning process.

\section{PARTICIPANTS AND PROCEDURES}

The participants for this study were selected from among 40 students enrolled in an upper-level introductory microbiology lab course. In the interest of this exploration, the selected criteria required the participation of average students. This would simplify the study thereby helping to identify the general issues and create a general understanding. As part of the course, all students were required to use Identibacter to identify several unknowns. The study participants were required to use the simulation in the research lab as they completed this assignment, whereas the other class members used the simulation in the university-sponsored computer lab. Selected students participated in a series of problem-solving sessions using Identibacter. During each session, students were videotaped as they interacted with the simulation.

For this study only five students were selected. Due to the research questions that we wanted to investigate, it was not appropriate nor feasible to study a large sample size. While generalizability is important, the purpose of this study was to explore the use of the software as a learning tool. The best way to accomplish this was to use a small sample size and to get detailed descriptions about the use of this tool. For future studies, we intend to automate the data collection process thereby permitting the extensive analysis of quantitative data.

\section{DATA COLLECTION AND ANALYSIS}

In trying to answer the research issues, this study used think-aloud protocol and video action logs (compiled to create a comprehensive report) as the principal methods to help answer the research inquiry. In order to create the video action logs, the researcher videotaped the computer screen as the participants used the simulation. Simultaneously, students verbalized their thinking as they worked through the problem-solving process of identifying an unknown. These verbal statements, in conjunction with the action logs, documented how students used the simulation and how the simulation components supported their problem-solving strategy development.

The analysis process included generating a coding scheme that accounted for the simulation components. The analysis codes accounted for the following questions. How do students use Identibacter? How did the students use the various features of the simulation? How do the students think as they problem solve? Do they construct effective problem-solving strategies using this simulation? The data were then coded and analyzed using qualitative analysis computer software in conjunction with spreadsheet software.

The research questions that were used to guide both the data collection and analysis are divided into two themes. Theme one, Simulation Usage, deals with how students used the simulation to practice bacterial identification. This theme 
investigated the components of the software that students used. Theme two, Problem-Solving Strategy Performance, focused on students' performance as they used Identibacter to practice solving multiple unknowns. Their performance was analyzed using four key expert-problem-solving-strategies characteristics. While this article points to the first theme, the second theme is described to give credibility to the use of Identibacter. The second theme forms the basis for most of the data interpretation.

Simulation usage analysis was based on the functional components of the simulation. The main analysis components included the Test Window, the Test Reference Window, and the Taxonomic Characteristics Window. The analysis investigated the various subcomponents within each window. Using the action logs, reports were generated that compiled the actions for each software component. For example, a report was generated that listed all of the tests that were run for each unknown. Action logs were also generated that indicated all of the various actions that a user took to identify an unknown. From the reports, simulation components usage was measured. Since students may have used the components for a variety of reasons, the usage was analyzed along with the details of the think-aloud protocols to look for variations in usage. Certain student performances merited further analysis. For example, one report was analyzed to delineate the identification accuracy and to categorize any errors. As appropriate for each report, averages, percentages, and ratios were calculated to compare the data. In addition, the action logs were used to calculate the time and percentage of total time that students spent using the various simulation components.

To analyze the second theme, student problem-solving performance, the progress reports and action logs were imported into a spreadsheet. To measure student performance, each unknown was analyzed based on four expert characteristics. They include: narrowing down the possible unknowns to a single group before narrowing down at the genus level, distinguishing between general and specific tests, accurately interpreting test results, and executing as few tests as possible. Based on student performance, three strategy levels were created with criteria for each level.

Lastly, all of the students were combined for cross-case analysis to determine how well the five participants performed as a group. Again, the four proficiency characteristics were used to measure performance. This illuminates the strategy development for the participants showing the learning that takes place and also how the simulation supports their learning.

\section{FINDINGS}

Simulation usage. The students used all of the functional components in the simulation. Some components were found to be more valuable than others. The students used the software components in several ways. The various components were used as: a reference source, a management tool (tracking groups and genera), a laboratory simulation (selecting, running, and interpreting diagnostic tests), and a problem-solving strategy simulation (narrowing down at the group and genus levels).

The five participants ran a total of 20 unknowns. Their combined time using the simulation was 332 minutes with an average of 16.6 minutes per unknown. Students ran a total of 250 tests with the average number of tests run per unknown at 12.5.

General use. Using the simulation, students generally followed a series of similar steps to solve bacterial unknowns. Having observed student performance, a definite pattern of use is evident (Fig. 1). Students first selected an unknown. After logging-in, reading the background information, and viewing the isolation medium plate, the user typically ran a few general or specific tests and interpreted the test results. Next they would exclude at either the groups, genera, or species level, and then they would identify the unknown.

Simulation components usage. Because of the unique nature of each window, a simulation window (component) was the unit of analysis for this study. Students used all of the software components during their participation in this study. However, some of the simulation components were used in varying degrees. Both the Taxon Characteristics Window and the Test Window primarily supported students in the bacterial identification problem-solving process. These two components were used $50 \%$ and $34 \%$ respectively for a combined total of $84 \%$ of the time that students used the simulation. The other components such as the Test Reference Window, the Species List Windows, and the Review Response Window were used as secondary support. Collectively, students used these components $2.2 \%$ of the total time. The Test Reference Window was used $13.8 \%$. So of all the various components that students used, the Taxon Characteristics Window was used the most.

Taxon Characteristics Window. Based on student usage, the most important simulation component helping students learn the process of bacterial identification was the Taxonomic Characteristics Window (Fig. 2). This component helped support problem-solving strategy development. Specifically, this component helps students to learn the processes of exclusion and of determining an appropriate test to run.

Generally speaking, one of the participants commented that the Taxonomic Characteristics Window "gave you a broad picture and it made you actually think about (the different characteristics)... because we always test different aspects of bacteria, but this...actually, let us put it all together, and figure out what we're doing with it." In a final interview, another participant commented, "I guess it organized my framework for starting from point A and then organizing a strategy for going through the identification that I probably wouldn't have had prior to it, prior to the program. I would qualify it as reinforcing my approach to (bacterial) identification.”

Having observed students, the process of exclusion is supported principally by the Taxon Characteristics Window. The functionality in this window allows the students to think in 
FIG. 1. General use and problem-solving strategy—showing relationships of components of software to the overall processes of identification.

FIG. 2. Taxon Characteristics Window showing group and genus level characteristics. 
terms of included and excluded organisms. In addition to providing taxon information, the functionality of this component was set up to provide a mechanism to facilitate the excluding process involved in bacterial identification. While the design is simple, this component simulates the organization and mechanism that an expert would use to problem solve.

Simply, the user is presented with three pop-up lists: a list to switch between the group or genus level information, a list of included taxons, and a list of excluded taxons. Initially, this window displays the group information and all unknown organisms are in the Included list. At any point, the user can exclude any item on the Included list. Likewise, any item on the Excluded list can be transferred to the Included list. Also, the user can view either the group or genera level information. If a student excludes a specific group, all of the associated genera that are included in that group are automatically excluded.

If more than one organism remains in the Included list, then the students continue the narrowing down process by selecting another test to run and then excluding all nonmatching organisms. Typically users continue this process until they have narrowed the list down to one species. If all the tests were interpreted correctly and the exclusion process was accurate, the remaining species is presumably the correct unknown.

Once the appropriate taxa have been excluded, the students then go though the process of determining the next test to run. This requires the student to compare the taxon characteristics and determine a common characteristic to distinguish among the remaining taxon candidates. Once a common characteristic has been determined, the student then runs the appropriate test.

Like the Test Window, the Taxonomic Characteristics Window is an integral part of the simulation, but it does not simulate the laboratory's physical environment. More than providing key taxon characteristics, the Taxonomic Characteristics Window was the key simulation component that allowed students to practice problem solving. Students used the Taxon Characteristics Window 50\% of the total time that they used this simulation. Considering all of the various components of the simulation, students spent a substantial amount of time working with the taxon information, excluding nonrelevant taxa, and determining the next test to run.

Students used all of the software components during their participation in this study. However, some components were used in varying degrees. The Test Window provided a mechanism for students to run the various tests, and the Test Reference Window provided information to help students interpret the test results. The Taxon Characteristics Window provided a means for managing all the unknown organisms, presenting group and genera characteristics, and facilitating the student's excluding strategy.

Problem-solving strategy performance. While students used the simulation in a similar manner, their performances varied. The second theme, Problem-Solving Strategy Development, addresses the overall student performance looking at elements of the deductive strategy, number of tests run, and interpretation of test results.

Based on the data analysis, four expert proficiency characteristics were generated (Fig. 3). The four characteristics include:

- Taxon Elimination Order (TEO)—This measure describes how well the participants narrowed down the possible unknowns to a single group before narrowing down at the genus and then species levels. The findings show that two of the five students showed proficiency for all of their runs. The other three students improved their performance over successive runs and demonstrated proficiency on their final run. Overall, students

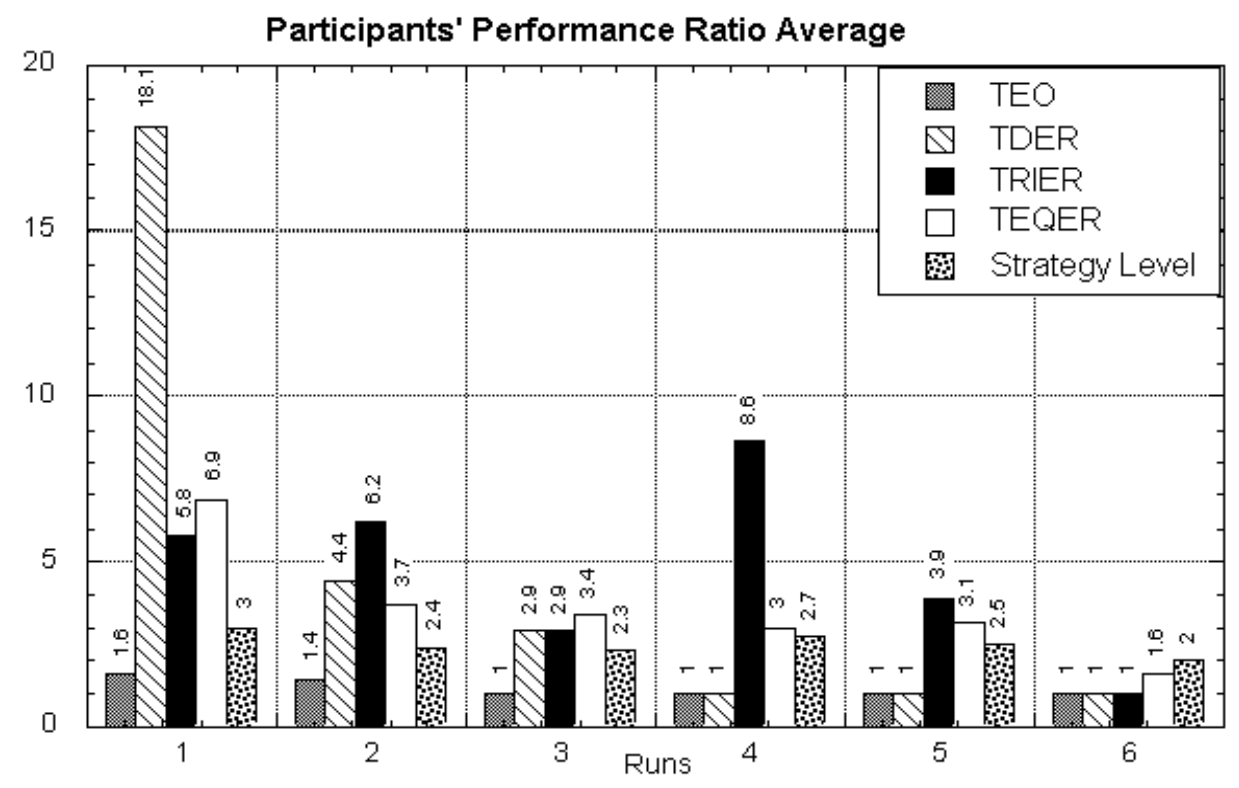

Fig. 3. Overall performance ratio-participants versus expert performance averages over six runs. 
quickly mastered this performance skill.

- $\quad$ Test Differentiation Error Rate (TDER) - This rate expresses the ability of students to distinguish between general and specific tests. There is a definite indication of TDER performance improvement over repeated runs for the participants in this study. For the students as a whole, this skill had the largest ratio. Students mastered this performance skill but not as fast as the TEO.

- Test Results Interpretation Error Rate (TRIER)—This ratio describes how accurately students interpret test results. From the findings, there are indications that test interpretation skills are not easily mastered, yet two of the five participants did not have any interpretation errors. For the students that had errors, interpretation performance improved so that for their final unknowns, all interpretations were accurate. Students were not as accurate as with other performance skills.

- Test Execution Quantity Efficiency Ratio (TEQER) The main objective of this measure was to determine if students were efficient with the amount of tests that they chose to run. The overall trend indicates that as students continue to run unknowns, they improve their ability to identify unknowns with fewer tests. This skill was the one that all of the students did not master, but their skill level was increasing during this study.

To determine the averages for the preceding characteristics, student performance was compared with expert performance. For example, the TRIER characteristic shows a value of 5.8 for the first run. This means that students performed 5.8 times the number of errors as compared with the expert for the same unknown. As scores approach 1, students are performing more like experts.

In this study we are looking specifically at cognitive performance measures. The measures (TEO, TDER, TRIER, and TEQER) look at students' use of the tool thereby considering their problem-solving strategy development. Assuming that students know how to navigate the program, the functionality of the program would not specifically affect these performance measures.

Based on the expert proficiency characteristics, problem-solving strategy performance levels were determined. Students in this study performed initially at the novice level and progressed to the intermediate level. None of the students performed at the expert level. The main categories are described as follows.

Expert level (score $=1$ ): Advanced users typically demonstrate the following performance characteristics: the ability to narrow down to a single group first, ability to distinguish between general and specific tests, ability to interpret test results correctly and use test data efficiently. Intermediate level (score $=2$ ): Intermediate users demonstrate all of the characteristics of an expert except they fail to use the test data efficiently. Novice level (score $=3$ ): Novices demonstrate one or more of the following characteristics: failure to narrow down to a single group first, inability to distinguish between general and specific tests, susceptibility to interpretation errors, and failure to use the test data effi- ciently.

\section{CONCLUSIONS}

Based on the data findings, there is evidence that students who use the simulation over successive runs improve their bacterial identification performance skills. As students use Identibacter to practice bacterial identification, the simulation supports their cognitive development by providing several key components during the learning process.

General instructional benefits. Identibacter affects many aspects of cognitive processing. Of significance, the simulation makes it possible for students to practice applying a domain-specific problem-solving strategy in a typical educational setting. The simulation becomes a tool that allows students to practice the cognitive skills required to solve an unknown. Use of the simulation removes limitations imposed by logistical and mechanical constraints that are found in a real microbiology laboratory. Further, the skills needed to run tests no longer occupy the student's attention, thereby helping students to quickly think through the process of solving unknowns.

Two general types of components that are beneficial for improving a student's problem-solving skills have become evident: (i) those that support the learning of a problem-solving strategy and (ii) those that support the learning process and provide the necessary reference tools in a single package so that students can focus on solving unknowns.

Components that support the learning of a problemsolving strategy. The components that support problem solving include both the Test Window and the Taxonomic Characteristics Window. The key component that impacts the learning of the problem-solving strategy is the Taxonomic Characteristics Window. From an instructional perspective, the Taxonomic Characteristics Window models the type of problem-solving that students are expected to learn and master.

The Taxonomic Characteristics Window models the key elements of a general identification strategy - where all of the incorrect unknowns are eliminated until only one organism is left. One of the expert characteristics is the ability to narrow down to one group prior to narrowing down at the genera level. The Taxonomic Characteristics Window facilitates the learning of this skill. The functionality of having different levels and different lists that are associated with each level make it difficult for students to jump back and forth as they are trying to identify organisms. A much simpler strategy would be to follow the functionality of the simulation and narrow down at each level before switching to the next more specific level. If students narrow down to one group first, they have much less information to sort through at the genera level.

Components that support the learning process. Learning support components include both the Test Window and the Taxonomic Characteristics Window. Principally the Test Window simulates the running of diagnostic tests. The less-used components such as the Species List and the 
Test Reference Windows were utilized but more for verbal information. While these features facilitated the learning process, they did not provide any support for helping students learn the key skills involved in bacterial identification.

The one expert characteristic that is not supported by Identibacter's functionality is the skill of running as few tests as possible. Because Identibacter facilitates running tests, students do not get the sense that running tests requires time and resources. Thus, Identibacter's design strength may encourage students not to think about issues of efficiency.

Skills developed with the use of the simulation. The simulation environment allows students to make their own decisions about what steps to perform as they apply their problem-solving strategy. Under these conditions, students are able to practice several skills. The key intellectual skills that students practice include:

- determining initial and subsequent tests,

- $\quad$ correctly interpreting tests, and

- excluding taxa.

All of these skills are critical to successfully identifying a microbial unknown.

Based on the problem-solving strategy performance levels, students did not perform at an expert level with respect to decision-making skills efficiency. This was the one reason why none of the participants reached the expert level. While this skill is desirable, efficiency was not one of the instructional goals for the laboratory setting in which this study was conducted.

The characteristics of this simulation that impact the learning of problem-solving strategies have been presented. This knowledge combined with the student performance data clarifies how Identibacter successfully helps students acquire higher-order thinking skills. The various simulation components support students' learning of bacterial identification.

In general terms, our findings have applications for educators of specific fields that are interested in increasing problem-solving skills using technology. The knowledge gained from this study may help instructional designers develop purposeful simulations that further enhance problem-solving skills. Further, educators can determine how a particular simulation is increasing their students' capabilities and perhaps provide critical support on occasions where a simulation may not be as effective as hoped.

\section{REFERENCES}

1. Farynaiarz, J. V., and L. G. Lockwood. 1992. Effectiveness of microcomputer simulations in stimulating environmental problem solving by community college students. J. Res. Sci. Teaching 29:453-470.

2. Gokhale, A. A. 1996. Effectiveness of computer simulation for enhancing higher order thinking. J. Ind. Teacher Educ. 33(4):36-46.

3. Jonassen, D. H., and T. C. Reeves. 1996. Learning with technology: using computers as cognitive tools, p. 693-719. In D. H. Jonassen (ed.), Handbook of research on educa- tional communications and technology. Macmillan, New York, N.Y.

4. Lieberman, D. A., and M. C. Linn. 1991. Learning to learn revisited: computers and the development of self-directed learning skills. J. Res. in Computing in Educ. 23:373395.

5. Rivers, R. H., and E. Vockell. 1987. Computer simulations to stimulate scientific problem solving. J. Res. in Sci. Teaching 24:403-415.

6. Salomon, G., and H. Gardner. 1986. The computer as educator: lessons from television research. Educ. Res. 15:1319.

7. Schank, R. C., and C. Cleary. 1995. Engines for education. Lawrence Erlbaum Associates, Hillsdale, N.J.

8. Thomas, R., and E. Hooper. 1991. Simulations: an opportunity we are missing. J. Res. in Computing in Educ. 23:497-513.

9. Thornburg, D. D. 1986. Restoring inductive reasoning. A+ :77-91.

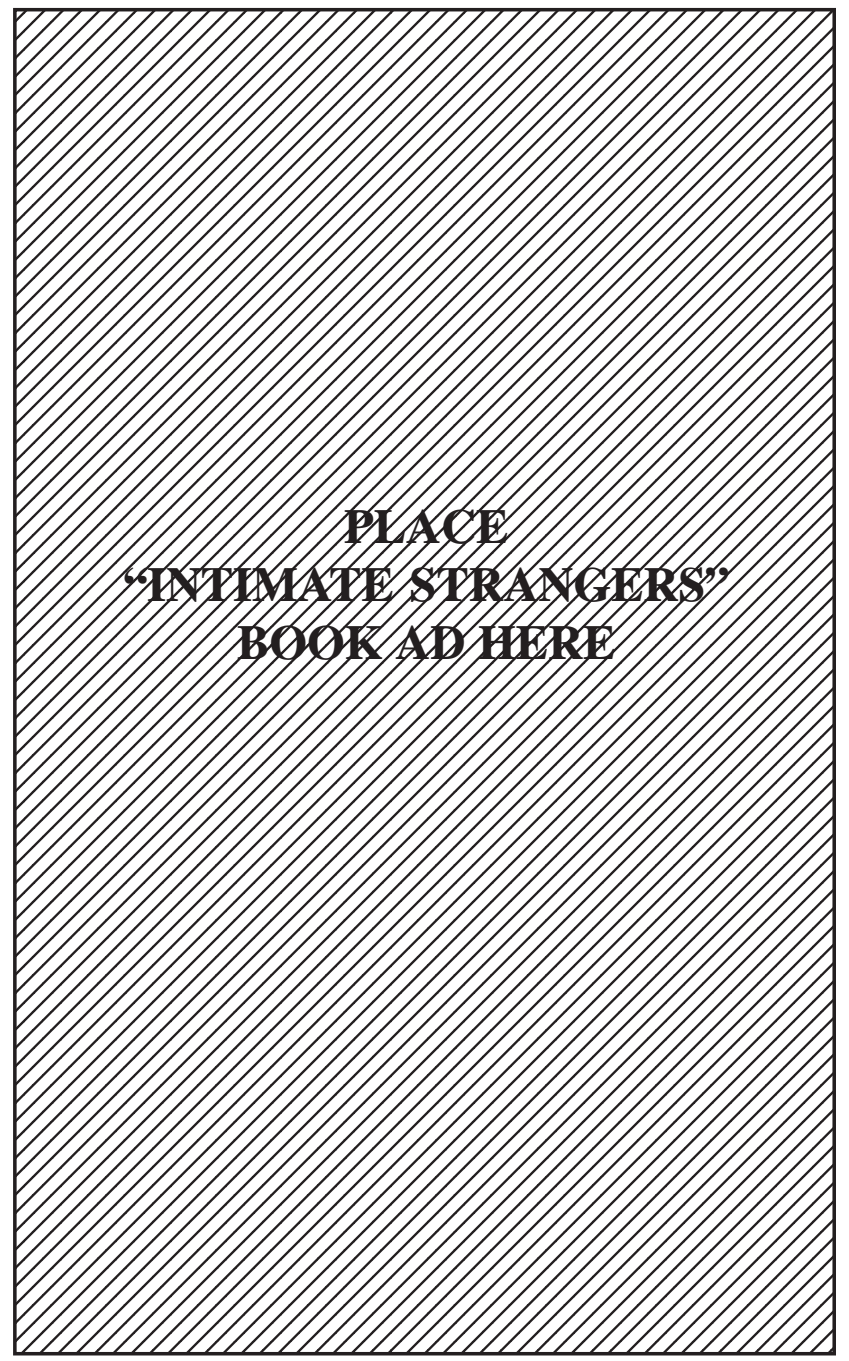

